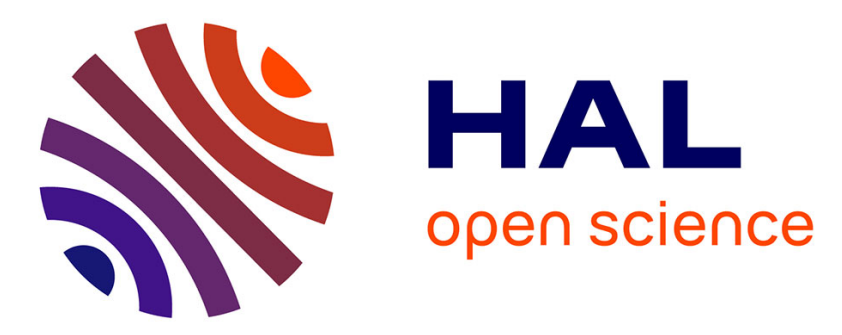

\title{
Random Calibration for Accelerating MR-ARFI Guided Ultrasonic Focusing in Transcranial Therapy
}

Na Liu, Antoine Liutkus, Jean-François Aubry, Laurent Marsac, Mickael Tanter, Laurent Daudet

\section{- To cite this version:}

Na Liu, Antoine Liutkus, Jean-François Aubry, Laurent Marsac, Mickael Tanter, et al.. Random Calibration for Accelerating MR-ARFI Guided Ultrasonic Focusing in Transcranial Therapy. Physics in Medicine and Biology, 2015, 60 (3), pp.21. 10.1088/0031-9155/60/3/1069 . hal-01104616

\author{
HAL Id: hal-01104616 \\ https://hal.inria.fr/hal-01104616
}

Submitted on 18 Jan 2015

HAL is a multi-disciplinary open access archive for the deposit and dissemination of scientific research documents, whether they are published or not. The documents may come from teaching and research institutions in France or abroad, or from public or private research centers.
L'archive ouverte pluridisciplinaire $\mathbf{H A L}$, est destinée au dépôt et à la diffusion de documents scientifiques de niveau recherche, publiés ou non, émanant des établissements d'enseignement et de recherche français ou étrangers, des laboratoires publics ou privés. 


\title{
Random Calibration for Accelerating MR-ARFI Guided Ultrasonic Focusing in Transcranial Therapy
}

\author{
Na Liu ${ }^{1}$, Antoine Liutkus ${ }^{1,2,3,4}$, Jean-François Aubry ${ }^{1}$, Laurent \\ Marsac $^{5}$, Mickael Tanter ${ }^{1}$, Laurent Daudet ${ }^{1}$ \\ ${ }^{1}$ Institut Langevin, UMR 7587, ESPCI ParisTech, CNRS, INSERM, Paris Diderot \\ University, 1 rue Jussieu, F-75005, Paris, France \\ ${ }^{2}$ Inria, Villers-lès-Nancy, F-54600, France \\ ${ }^{3}$ Université de Lorraine, LORIA, UMR 7503, Villers-lès-Nancy, F-54600, France \\ ${ }^{4}$ CNRS, LORIA, UMR 7503, Villers-lès-Nancy, F-54600, France \\ ${ }^{5}$ SuperSonic Imagine, Les jardins de la Duranne, Bât E, 510 Rue René Descartes \\ 13857, Aix en Provence, France \\ E-mail: na.liu@espci.fr
}

\begin{abstract}
Transcranial focused ultrasound is a promising therapeutic modality. It consists in placing transducers around the skull and emitting shaped ultrasound waves that propagate through the skull and then concentrate on one particular location within the brain. However, the skull bone is known to distort the ultrasound beam. In order to compensate for such distortions, a number of techniques have been proposed recently, for instance using Magnetic Resonance Imaging (MRI) feedback. In order to fully determine the focusing distortion due to the skull, such methods usually require as many calibration signals as transducers, resulting in a lengthy calibration process. In this paper, we investigate how the number of calibration sequences can be significantly reduced, based on random measurements and optimization techniques. Experimental data with six human skulls demonstrate that the number of measurements can be up to three times lower than with the standard methods, while restoring $90 \%$ of the focusing efficiency.
\end{abstract}




\section{Introduction}

Image-guided focused ultrasound (FUS) is an innovative non-invasive treatment option which does not involve ionizing radiation [8]. It has shown clinical success in the treatment of uterine fibroids [7, 17, 38], prostate cancers [29], liver tumors [44, 18], and bone tumor pain palliation $[15,23,30]$. Recently it has led to promising results in neurological diseases (neuropathic pain [19], essential tremor [5, 24]). As for the applications of brain therapy, it has for a long time been challenging to get the ultrasound focused with high quality on the targeted location inside the brain because of aberrations both in amplitude and phase caused by the heterogeneities of human skulls and brain tissue, in terms of ultrasound speed discrepancies (about $3000 \mathrm{~m} / \mathrm{s}$ in the skull and 1500 $\mathrm{m} / \mathrm{s}$ in brain tissue [9]), density, and ultrasonic attenuation [33]. These heterogeneities cause the distortion of the ultrasonic wave fields and thus can result in a partial destruction of the focusing pattern in the brain. Furthermore, it is also difficult to get the backscattered signals from the brain tissue as a result of the strong attenuations from the skull [33], which limits the use of Green's functions in clinical applications, which requires echoes from a bright reflector or a point-like active source located inside the biological tissues.

For non-invasive brain treatments, a remaining challenge is beam shaping to compensate for the skull-induced distortion [8]. Various adaptive techniques have been proposed, such as phase conjugation [16, 39], time-reversal [32, 42], and inverse filtering $[40,1]$. In clinical practice, this problem, which is central in transcranial focused ultrasound therapy, is currently addressed by using acoustic modeling based on a full head scan of the patient. Computed tomography (CT, [2, 25]) or magnetic resonance (MR, [16]) images of the skull bone can be used as inputs for the simulation schemes. These estimates are then used in the compensation process before the HIFU treatment. The feasibility of using image-derived skull thickness information for phase correction has been investigated by Hynynen et al. [16]. The details of the internal structures of the skull provided by CT images [4] have been shown to improve the focusing at high frequency [2]. However, this approach is limited both in terms of the physical/numerical models and by the computational cost of the high-frequency corrections. Furthermore, the patient is subjected to a significant radiation dose during the full head scan.

To overcome these limitations, novel focusing techniques, called "Energy-based focusing," have been developed, which rely on the indirect estimation of the acoustic wave intensity at the target location, for different coded excitations [13, 22]. Noninvasive implementations of these techniques are based on the estimation of the acoustic radiation force using MR imaging [37, 27]. MR acoustic radiation force imaging (MRARFI) indeed allows an indirect measurement of the amplitude of the acoustic pressure in situ, by encoding the tissue displacement induced by the acoustic radiation force into the phase of the MR signal [27, 14]. Therefore, maximizing the phase shift of the MR-ARFI signal corresponds to optimizing the acoustic energy deposition at focus. MRARFI guided ultrasonic focusing is of great interest, as it is based on the experimental 
data alone, without any prior model on the propagation medium [22]. However, the main intrinsic limitations of this approach are the low SNR of the MR signal, and the high number of test sonications needed. As the radiation force is proportional to the beam energy at focus, at least three de-phased sets of sonications are necessary to estimate the relative phase of each ultrasonic beam, by the so-called phase-stepping techniques $[22,20,43]$. For an array with $N$ elements, at least $3 N$ ultrasonic sonications are then required for the full array calibration, usually employing a Hadamard basis. As the number of elements in therapeutic arrays typically ranges from 512 to 1024 [3, 6], the whole calibration process currently takes hours [26], with a high cumulative energy deposition, proportional to the number of shots. It is therefore essential to reduce the time for the calibration of the focusing, especially for stroke treatments, which require a fast therapeutic response ( $<4 \mathrm{~h}$ after the event) [28].

To optimize the time for acquisition and calibration, Kaye et al. have proposed an acceleration of the adaptive focusing process by replacing the Hadamard basis [22] by Zernike polynomials (ZPs) [20]. It must be noted that both the Hadamard basis and the ZPs have constraints: the order of a Hadamard basis must be 1,2 or a multiple of 4 , and the technique using Zernike polynomials requires the precise coordinates of the elements of the array, which is proprietary information in some brain therapy systems. Furthermore, the order of the ZPs must be large enough to take into account the discrepancies between different human skulls.

The purpose of this paper is to propose a novel scheme for encoding the sequence of test sonications in order to reduce their number, while maintaining an adequate focusing performance. In the first part of this paper (Section 2), we introduce the free-field propagation theory and the concepts of virtual transducers, where the skull is modeled as an infinitely thin scattering material between the ultrasound arrays and the virtual transducers. A new algorithm for calibration is then introduced (Section 3), based on least-squares minimization, allowing the use of random calibration sequences. In the second part of this paper (Section 4), the results from both simulations and real experiments based on six human skulls are presented, to test the validity of this new calibration method. This is done by comparing the focusing quality at the target location as a function of the number of calibration measurements, for different calibration sequences (Hadamard, ZPs, random).

\section{Background and theory}

\subsection{Background}

During an MR-ARFI transcranial focusing experiment, the total acoustic force at the focusing location is the sum of all the responses caused by all individual active ultrasound transducers. Throughout this study, we assume that $N$ transducers are available and that their relative positions remain fixed during the procedure. The signal emitted by transducer $n$ at time $t$ is denoted $\tilde{x}_{n}(t)$. We are especially interested in the resulting 
acoustic field $\tilde{y}_{m}(t)$ at a set of $M$ distinct spatial positions within a region of interest (ROI). In the original studies by Tanter et al. [41, 40, 1]), the signal observed at $\tilde{y}_{m}(t)$ in the ROI $(m=1 \ldots M)$ is modeled in a linear setting as the superposition of filtered versions of the inputs $\tilde{x}_{n}(t)$ :

$$
\tilde{y}_{m}(t)=\sum_{n=1}^{N} p_{m n}(t) * \tilde{x}_{n}(t),
$$

where $*$ denotes the convolution product, and $p_{m n}(t)$ is the impulse response accounting for the acoustic path from transducer $n$ to output point $m$. It notably encompasses both the influence of the free-field propagation and aberrations due to the presence of the skull between the transducers and the ROI. In our experimental setup, we have $M>N$. Indeed, the usual HIFU devices typically feature $N \sim 500$ transducers and the ROI is divided into $M \sim 10^{4}$ individual cubic elements of size $\lambda / 4$. In the steady-state case, (1) can be written in the frequency domain [21] as

$$
Y_{m}(f)=\sum_{n=1}^{N} P_{m n}(f) X_{n}(f),
$$

or, more concisely,

$$
y(f)=P(f) x(f),
$$

where $y(f)$ and $x(f)$ are $M \times 1$ and $N \times 1$ vectors and $P(f)$ is an $M \times N$ matrix, called the Transmission Matrix (TM). Their entries $[x(f)]_{m},[y(f)]_{n}$ and $[P(f)]_{m n}$ correspond, respectively, to the Fourier transforms of $\tilde{x}_{n}, \tilde{y}_{n}$, and $p_{m n}$ at frequency $f$.

In this study, we decompose the complete TM $P$ as the product of two other matrices $P_{0}$ and $A$ of respective dimensions $M \times N$ and $N \times N$, by

$$
P(f)=P_{0}(f) A(f),
$$

where $P_{0}(f)$ is called the free-field $T M$ at frequency $f$ and $A(f)$ is called the apparent gain matrix at frequency $f$. The rationale underlying this decomposition is to decouple the influence of the skull from that of the acoustic free-field transportation. Whereas $P_{0}$ may be computed or measured beforehand $\ddagger$, only $A$ depends on the patient's skull. Since $M>N$, this decomposition is advantageous because it only leaves $N^{2}$ parameters to estimate instead of the potentially much larger $M \times N$. Combining (2) and (3), we get

$$
y=P_{0} A x .
$$

As can be seen, model (4) may be understood as $y=P_{0} z$, where $z$ gathers the signals emitted by virtual transducers, given by $z=A x$. This gives a better understanding of the role of the gain matrix $A$, which models the aberrations in the acoustic path as $\ddagger$ Working at a single frequency $f$, we drop the dependence with respect to $f$ in the notations for conciseness. 

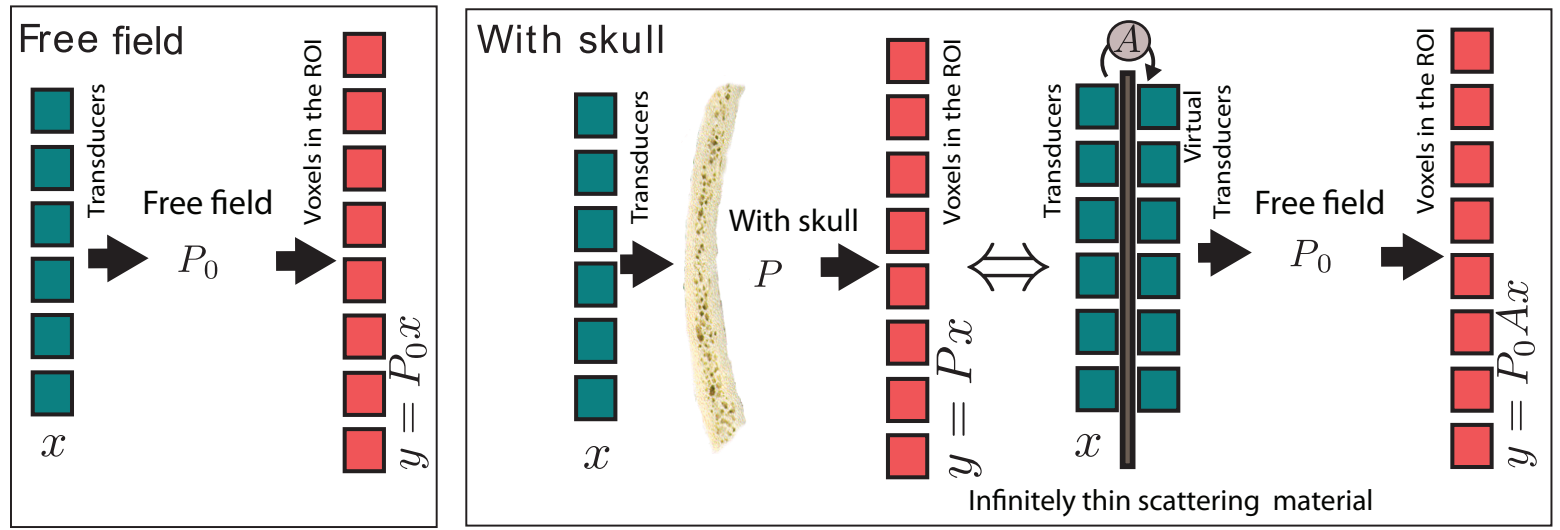

Figure 1. Proposed acoustic modeling. All aberrations are modeled as the action of an infinitely thin scattering material located in the transducer plane. Acoustic transportation to the region of interest is then modeled using the free-field transmission matrix.

modifications of the input signal itself. Put otherwise, all aberrations are modeled as occurring in an infinitely thin but highly scattering material located at the same place as the transducers $x$, yielding virtual transducers $z$. Then, propagation to the ROI is the same as in a free-field. In that sense, $A_{n n^{\prime}} \in \mathbb{C}$ encodes the gain from transducer $n^{\prime}$ to the virtual transducer $n$. It should be noted that we do not assume a localized model, where only transducer $n^{\prime}$ influences the virtual transducer $n$ at the same position, which would mean a diagonal matrix for $A$. Instead, $A$ is taken to be a full matrix, where non-local and even large-scale effects can be taken into account. The whole model is depicted in Fig. 1.

\subsection{Calibration of the transmission matrix}

In practice, the total transmission matrix $P$ in (2) is unknown and should be determined through experimental calibration. For this purpose, the practitioner can control the input signals $\tilde{x}_{n}(t)$, and measure the corresponding acoustic field $\tilde{y}_{m}(t)$. Then $P$ is estimated as the best linear mapping between $x$ and $y$.

A straightforward calibration technique consists in activating only one transducer $n$ $(1 \leqslant n \leqslant N)$ for each measurement, in order to acquire the resulting output displacement. Since only one transducer is active at a time, the output directly corresponds to the corresponding column of $P$. All columns are then measured sequentially. This calibration method however raises two issues. First, the signal to noise ratio (SNR) may not be advantageous, because each transducer can only emit with a limited sonication power. Second, the whole process requires a large number $N$ of measurements: one for each transducer.

Recently, it has been shown that these two limitations of the calibration process may be alleviated to some extent. In particular, Larrat et al. [22, 20] have demonstrated that it is not necessary to consider each transducer sequentially during the calibration process. 
Instead, several transducers may be activated at the same time, and the TM is then estimated with better precision. The main steps in this technique can be summarized as follows.

First, a set of inputs is chosen, which is an $N \times K$ matrix $X$. Each column of this matrix corresponds to a different set of $N$ complex values for the transducers, in the frequency domain (amplitude and phase). In the baseline method described above, we simply have $K=N$ and $X$ is taken as the identity matrix (the canonical basis): we activate only one transducer at a time, emitting a steady sinusoidal signal at full power. In the paper of Larrat et al. [22], $K$ is also set to $K=N$ and $X$ is chosen as a Hadamard matrix, which contains only the amplitude 0 or 1 , meaning that different sets of transducers are activated at full power for each measurement. In the paper of Kaye et al. [20], $K \leq N$ and $X$ is chosen as a set of Zernike polynomials. The rationale for this choice is that these $K$ vectors are more likely to correspond to some eigenvectors of $P$ than elements derived from the Hadamard basis.

Second, the corresponding output displacements are measured, yielding the $M \times K$ matrix $Y$ :

$$
Y=P X
$$

Finally, $P$ is estimated in the least-squares error sense by

$$
\hat{P}=Y X^{\dagger}
$$

where $\cdot^{\dagger}$ denotes pseudo-inversion. Similarly, several other studies in the literature have exploited this line of thought. In particular, recent studies in optics [43, 36] measure such transmission matrices with multiplexed input vectors. Whenever $K=N$, this strategy gives an accurate estimate of $P$. When $K<N$, the quality of the estimation depends on how well $X$ spans the eigenvectors of $P$.

In this study, we assume that the free-field TM $P_{0}$ is known. This matrix does not depend on the particular aberrations due to the skull, but rather on the geometrical configuration of the array of transducers. Hence, the calibration of $P_{0}$ may be done once and for all for a given array, before its clinical use. Then, we show that the gain matrix $A$ can also be estimated effectively through a random calibration design, i.e., by choosing a random $X$. This choice is motivated by the fact that approximate singular value decompositions of rank-deficient matrices can be obtained using only a limited number of matrix-vector evaluations [12]. In the same vein, we show here that only a few measurements are required to fully characterize $A$ if only $K<N$ of its singular values are non-negligible, which is verified in practice, as shall be demonstrated in the experimental part of this paper. The simplest design of the random matrix $X$ is by choosing independent random entries in $\{0,1\}$ with equal probability, i.e., a Bernoulli distribution, which in practice means that for a given measurement (column of $X$ ), the transducers are either off or on with full power, with equal probability. Other choices are independent random entries in $\{-1,1\}$ (transducers all on with full power, in phase or with a $180^{\circ}$ phase shift, with equal probability), or in $\{z \in \mathbb{C}|| z \mid=1\}$ (transducers all 
on with full power, with random phases uniformly chosen in $(0,2 \pi))$. The gain matrix $A$ is then estimated by

$$
\hat{A}=P_{0}^{\dagger} Y X^{\star}\left[X X^{\star}+\sigma^{2} \mathbf{I}_{M}\right]^{-1}
$$

where $\cdot^{\star}$ denotes complex conjugation, $\sigma$ is a regularization constant related to the noise level, and $\mathbf{I}_{M}$ denotes the $M \times M$ identity matrix. Equation (6) may be derived in the least-squares error sense when the noise in the observations is assumed Gaussian, independent and identically distributed (i.i.d.), with variance $\sigma^{2}$. Alternatively, $\sigma^{2} \mathbf{I}_{M}$ may simply be seen as a Tikhonov regularization term.

As can be seen, this procedure only involves basic algebraic manipulations and does not require the delicate computation of input configurations that depend on the geometry of the transducers array, as in the case of the ZPs.

\subsection{Focusing}

Given the TM $P=P_{0} A$ estimated by the above procedure, the objective is now to shape the signal emitted by the transducers so as to focus within the ROI. For this purpose, we get back to the forward model (2). In practice, some noise is bound to be present in the process, yielding

$$
y=P_{0} A x+\epsilon,
$$

where $\epsilon$ is an $M \times 1$ vector accounting for noise, whose covariance matrix is assumed diagonal: $\mathbb{E}\left[\epsilon \epsilon^{\star}\right]=\sigma^{2} \mathbf{I}_{M}$. So as to proceed to focusing, we define a target output $\sigma^{2} y_{t}$, where $y_{t}$ is identically 0 except for the target points that are set to 1 . If $\sigma^{2}$ is known, our objective becomes the estimation of the corresponding input $\hat{x}_{\mid \sigma}$ that yields an output as close as possible to $\sigma^{2} y_{t}$. This can be achieved in the minimum mean-squared error (MMSE) sense by choosing

$$
\hat{x}_{\mid \sigma}=\underset{x}{\operatorname{argmin}}\left\|\sigma^{2} y_{t}-P_{0} A x\right\|_{2},
$$

which is readily shown to be equal to

$$
\hat{x}_{\mid \sigma}=\left(P_{0} A\right)^{\star}\left[P_{0} A A^{\star} P_{0}^{\star}+\sigma^{2} \mathbf{I}_{M}\right]^{-1} \sigma^{2} y_{t} .
$$

When the power $\sigma^{2}$ of the noise tends to infinity, it is readily shown that (8) is equivalent to

$$
\lim _{\sigma \rightarrow \infty} \hat{x}_{\mid \sigma}=\left(P_{0} A\right)^{\star} y_{t}
$$

so that $\hat{x}_{\mid \infty}$ is simply estimated through the Time-Reversal Mirror (TRM) [41, 40, 1]. TRM is indeed a popular way of focusing waves through disordered media, mainly thanks to its robustness to noise. As we see here, this robustness property may be understood on probabilistic grounds: TRM is the optimal MMSE estimator as the energy of the noise becomes infinite. Its efficiency for transcranial ultrasound therapy has been demonstrated [10]. 

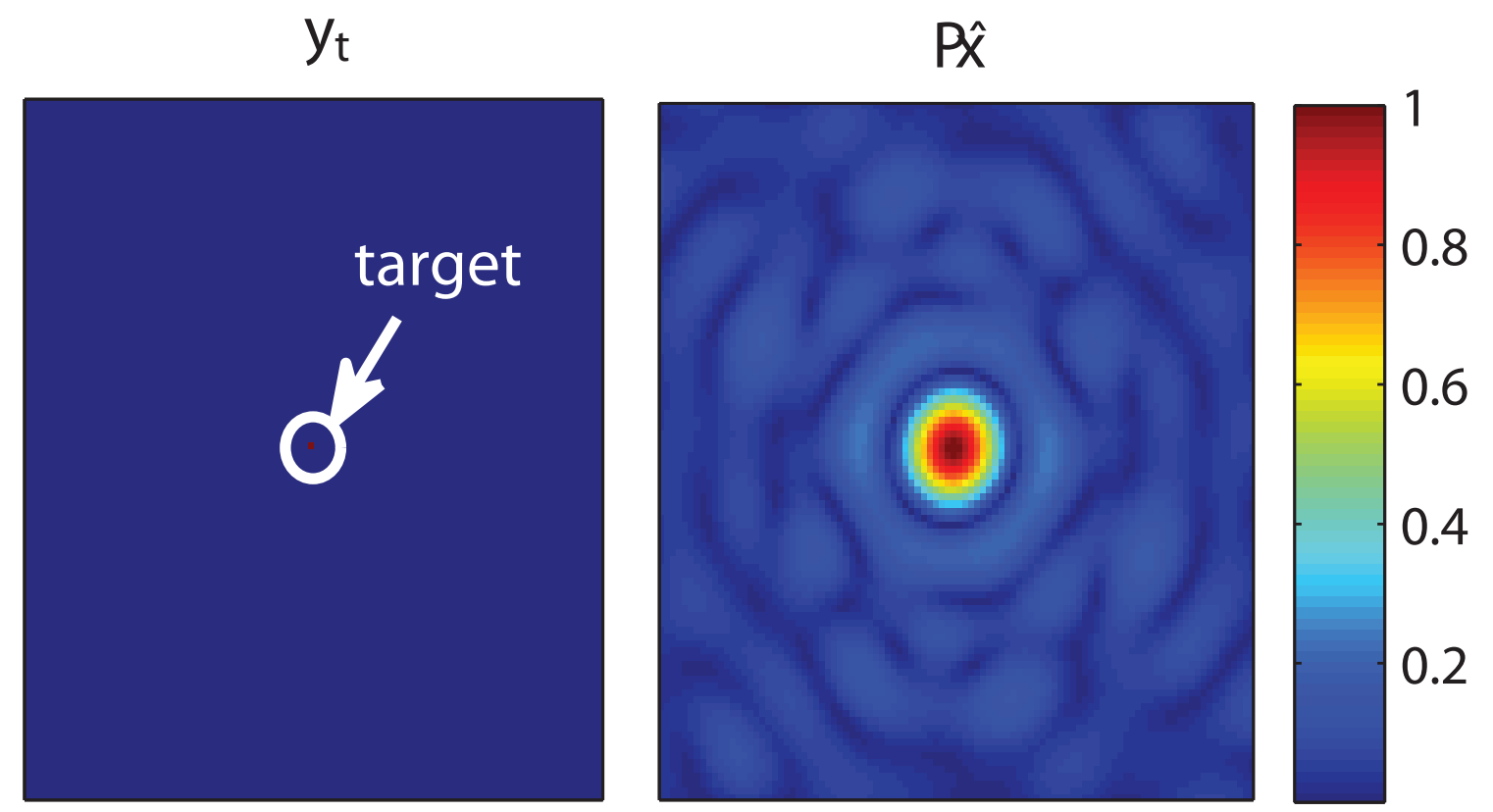

Figure 2. Synthetic example of focusing with the Time Reversal Mirror. Left: targeted signal output $y_{t}$. Right: observed output $P \hat{x}$ where $\hat{x}$ is estimated by using (10). $P=P_{0} A$ is drawn randomly and perfectly known.

If an estimate of the noise power $\sigma^{2}$ is available or if the noise is small, using the complete expression (8) will yield better performance, as for instance demonstrated in an optical context in [35]. In any case, we will simply write

$$
\hat{x}=\left(P_{0} A\right)^{\dagger} y_{t}
$$

where $\cdot{ }^{\dagger}$ denotes either complex conjugation if TRM is chosen or Moore-Penrose pseudoinversion otherwise [40].

In this paper, time reversal is used as the gold standard for aberration correction: the pressure obtained at the target for each focusing technique is compared to time reversal (time reversal corresponds to 100\%). In Fig. 2, we show an example of the typical focusing performance of TRM in ideal conditions, i.e., when the TM $P=P_{0} A$ is perfectly known.

\section{Methods}

\subsection{Simulations using synthetic data}

We first numerically investigate the focusing performance when the free-field TM $P_{0}$ is known and $A$ is estimated using $K \leq N$ calibration measurements. For this purpose, $P_{0}$ is set to the theoretical free-field TM corresponding to the actual positions of the transducers of our HIFU equipment [26] and $A$ is generated with independent and 
identically distributed (i.i.d.) complex centered Gaussian entries, corresponding to highly scattering aberrations $[11,36]$. We here set $M=10^{4}$ and $N=512$.

The calibration algorithm described in the previous section was applied with different numbers $K$ of calibration measurements, using random input vectors $X$ drawn from a Bernoulli distribution with equal $\{0,1\}$ probability. In order to simulate the effect of measurement noise, the observations were modelled by $Y=P_{0} A X+\epsilon$, where the variance of $\epsilon$ was set to $3 \%$ of the energy of the clean output signal. Subsequently, $Y$ was used to compute $\hat{A}$ using (6). For a given number $K$ of calibration signals, the $\hat{A}$ obtained in this way was used to focus at a given location (the target $y_{t}$ was set so that one point in the ROI had 1 , and the other points have 0 ). The corresponding input $\hat{x}$ was estimated as in (10). The resulting output was finally constructed as $P \hat{x}=P_{0} A \hat{x}$.

\subsection{Analysis of real data and focusing experiments}

3.2.1. Experiment set up Six skulls were obtained from the Institut d'Anatomie UFR Biomédicale des Saints-Pères Université René Descartes in Paris. The specimens satisfied the criteria of the Centre du Don des Corps and all the donors gave their informed consent before death. Before any measurements were made, the skulls were degassed in water under constant low pressure for 24 hours.

A picture illustrating the experiment is shown in Fig. 3. The $1 \mathrm{MHz} \mathrm{HIFU}$ brain therapy probe (Imasonic, Voray sur l'Ognon, France) is composed of 418 active elements disposed on a portion of a sphere. Each element has an active diameter of 6 $\mathrm{mm}$, and a bandwidth of $800 \mathrm{kHz}-1.3 \mathrm{MHz}$. The probe was attached to a degassed water tank using a mechanical system, on which each skull was positioned with a stereotactic frame. The pressure field emitted by the elements of the probe was measured by a needle hydrophone (HCN400 model, Onda Corporation, Sunnyvale, CA, active diameter of $0.4 \mathrm{~mm}$ ) translated around the geometric focus of the probe. Motorized 3 -axis orthogonal translations axes allowed the measurement at different points within a volume of $6 \times 6 \times 4.5 \mathrm{~mm}^{3}$. The probe was controlled by a brain therapy electronic system (developed by SuperSonic Imagine, Aix en Provence, France) and programmed by a Matlab interface. It also acquired the signal measured by the hydrophone, so that the volume measurements could be automated. The signal frequency was centered on $1 \mathrm{MHz}$, and the experimental measurement resolution was set to $\lambda / 4$ in the lateral direction and $\lambda / 2$ in the axial directions.

Each TM was obtained by measuring the transmitted signal from each probe element to the hydrophone, and repeating this for each point of a 3D grid centered on the geometrical focus of the probe. The TM in water without the skull was acquired before each TM measurement through the skull. One TM was acquired for each skull and was then composed of the three spatial dimensions, one temporal and one probe element dimension. By taking a Fourier transform, the temporal dimension was reduced to a single complex value (amplitude and phase) at the center frequency. 


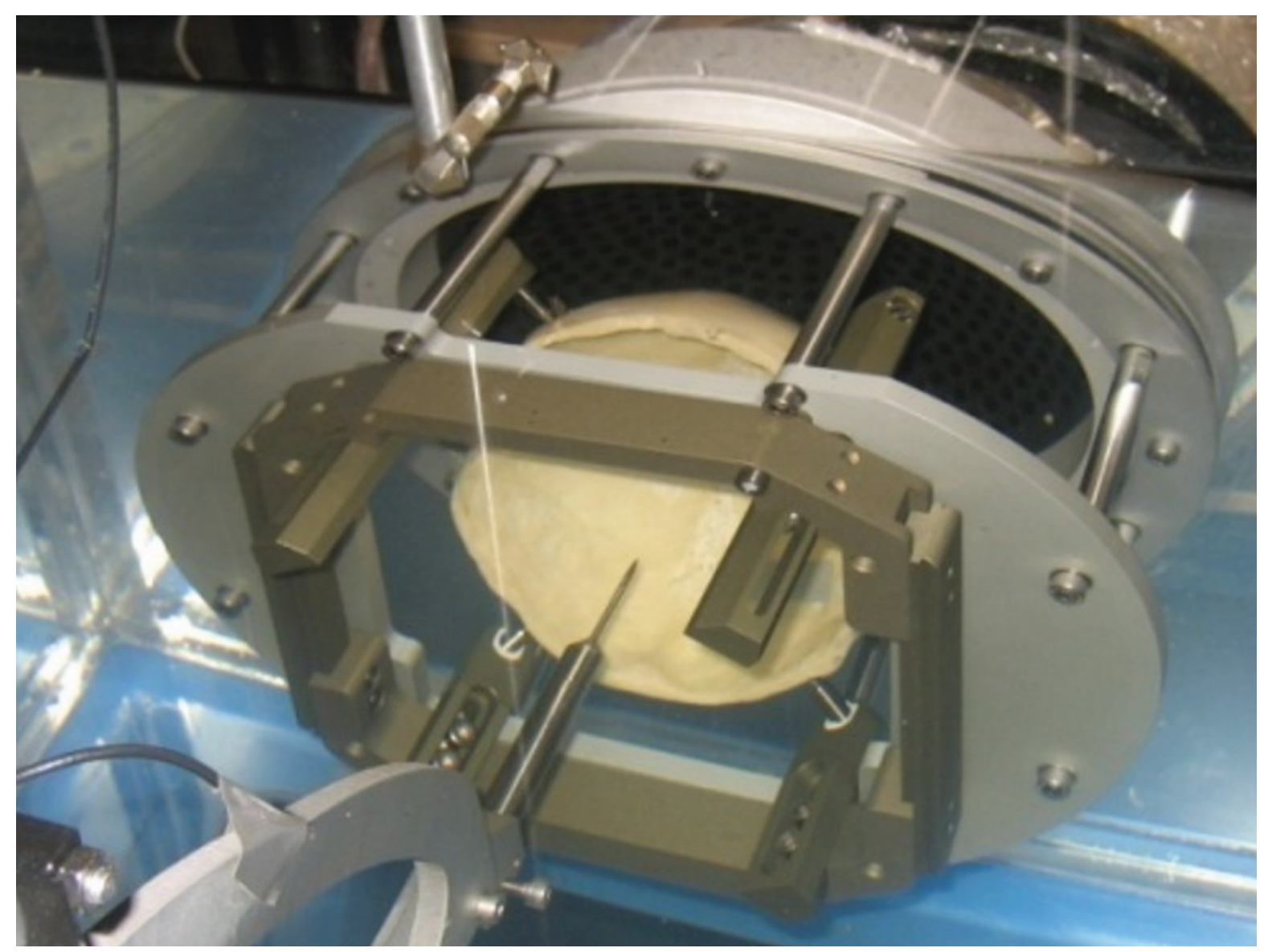

Figure 3. Picture of the experiment showing the skull mounted on a stereotactic frame in front of the probe, with the hydrophone placed at the probe's geometrical focus.

3.2.2. Experimental gain matrices For the $S=6$ human skull dataset, we can estimate the corresponding gain matrices $A^{(s)}$ by

$$
\forall s=1, \ldots, S, P^{(s)}=P_{0} A^{(s)},
$$

and $A^{(s)}$ was thus readily estimated in the sense of least-squares errors by $A^{(s)}=$ $P_{0}^{\dagger} P^{(s)}$. where ${ }^{\dagger}$ denotes complex conjugation. Further insights about the properties of the experimental gain matrices $A^{(s)}$ are gained by computing their Singular Value Decomposition (SVD).

3.2.3. Focusing In this section, we assume that the true $\mathrm{TM} P^{(s)}$ are known and, for a given input signal $x$, we simulate the output signal through the skull $(s)$ by $P^{(s)} x$, as in $[26]$.

The goal of this experiment was to compare several calibration techniques, as a function of $K$, the number of calibration measurements. These techniques only differ in the set of input signals $X$ used for calibration:

(i) A random i.i.d. draw from $X_{n k} \in\{0,1\}$, with equal probability. In this 
configuration, in one measurement, the elements of the array were either on at full power, or off, with equal probability.

(ii) A random i.i.d. draw from $X_{n k} \in\{-1,1\}$, with equal probability. Here, in one measurement, all elements in the array were on at full power, but with a $50 \%$ chance of a $180^{\circ}$ phase shift.

(iii) A random i.i.d. draw from $X_{n k}=\exp i 2 \pi \theta$, with $\theta$ uniformly distributed on $(0,2 \pi)$. All elements in the array were on at full power, each with a random phase (different for each measurement).

(iv) $K$ randomly chosen columns of the identity matrix (canonical basis). Here, only one element of the array was on for each measurement, with full power.

(v) The first $K$ columns of the $\{-1,+1\}$ Hadamard matrix [22]. Here, in one measurement, all elements in the array were on at full power, one-half of them with a $180^{\circ}$ phase shift, according to a Hadamard sequence.

(vi) The first $K$ Zernike polynomials, as described in [20]. Here, the elements were all given varying amplitudes according to a spatial model given by the Zernicke polynomials. It should be emphasized that this is the only calibration method tested here that requires an explicit knowledge of the positions of the transducers.

(vii) The first $K$ input eigenvectors of the full gain matrix A, assumed to be known. Although $A$ is not known in practical calibration setups, this "oracle" reference method gives an upper bound on the performance that can be achieved within this framework.

For a given choice of calibration sequence $X$, with $K$ columns (each column corresponding to one measurement), we simulated the corresponding measurements through the skull $s$ by $Y=P^{(s)} X$. This yields an estimate $\widehat{P^{(s)}}$ of the TM that uses only $P_{0}$ (assumed known), $X$, and $Y$. This estimate was finally used to simulate the focusing pattern on 100 different voxels in the ROI§. The performance was measured as the loss in power at focus induced by the use of $K<N$ calibration measurements, as compared to using the true TM $P^{(s)}$. The results were averaged over the $S=6$ different skulls.

\section{Results}

\subsection{Simulations on synthetic data}

The simulation results are displayed in Fig. 4. As can be seen, the proposed approach maintains a good focusing performance using only a fraction of the $N=512$ calibration measurements required by the baseline approach. These simulations suggest that the method may be applied to drastically reduce the number of calibration measurements.

$\S$ For one target $y_{t}$, the input was chosen through TRM as $\hat{x}={\widehat{P^{(s)}}}^{\star} y_{t}$ as in $(9)$. 


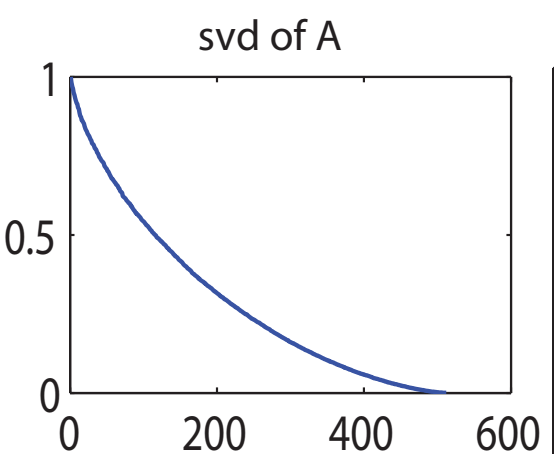

$\mathrm{K}=15$

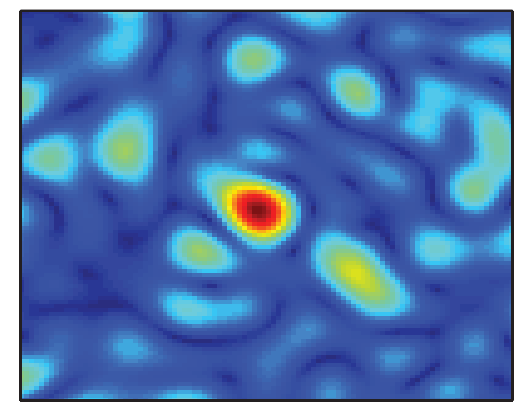

$\mathrm{K}=50$

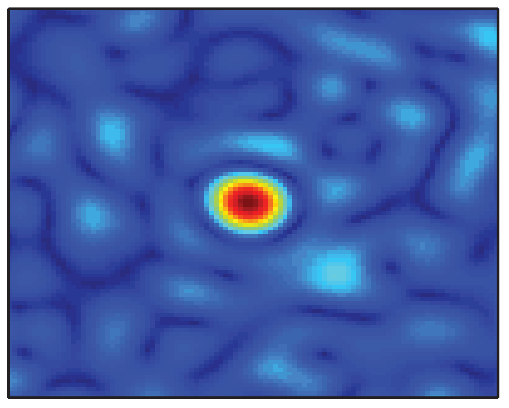

$\mathrm{K}=5$

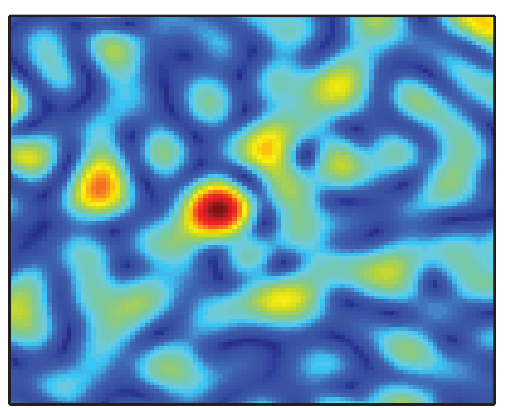

$\mathrm{K}=20$

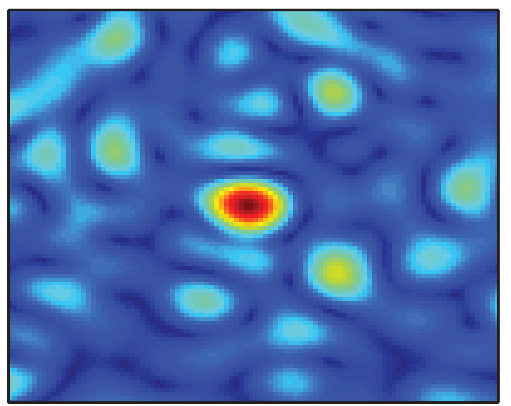

$K=100$

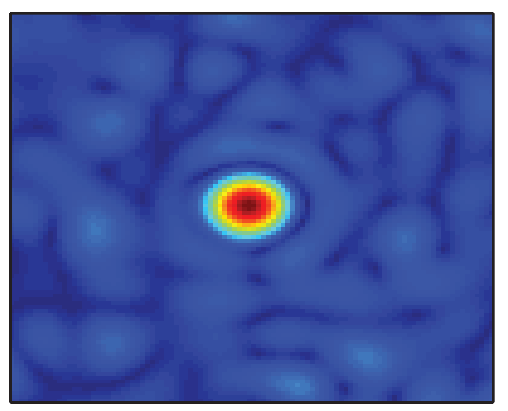

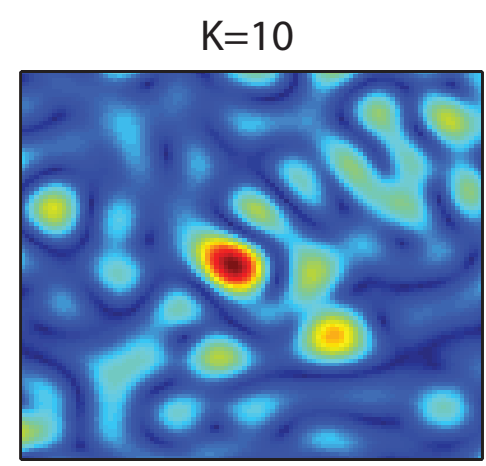

$\mathrm{K}=25$

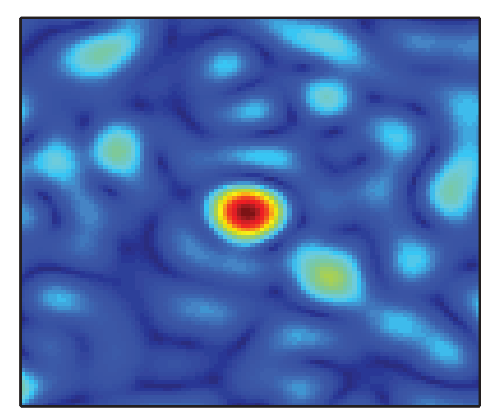

$\mathrm{K}=512$

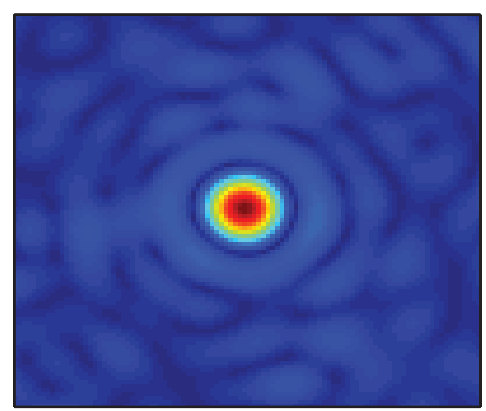

Figure 4. Focusing performance of the proposed method on simulated data with a varying number $K$ of calibration measurements. The number of transducers was $N=512$, and the simulations include a $3 \%$ noise power. Upper left: singular value decomposition of the true $A$ matrix to estimate. Plots are normalized.

\subsection{Analysis of experimental data and focusing experiments}

The experimental data consists of the complete TM $P^{(s)}$, acquired experimentally from the $S=6$ different human skulls $(s=1, \ldots, 6)$, and in the corresponding free-field TM $P_{0}$. The focusing ROI was a volume composed of $M=17 \times 17 \times 7=2023$ voxels and the number of active transducers was $N=418$. The data also comes with the exact spatial position of each transducer.

\subsubsection{Experimental mixing matrices A display of the resulting gain matrices is given in}

Fig. 5. As can be seen, three strong diagonals appear, indicating that the signal emitted by each transducer was mostly scattered to its two closest neighbors. Furthermore, strong vertical and horizontal structures are clearly visible and demonstrate that the 


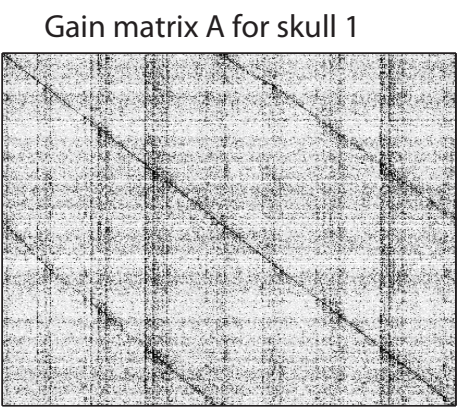

Gain matrix A for skull 4

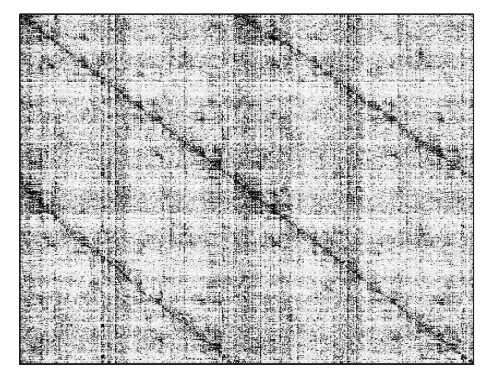

Gain matrix A for skull 2

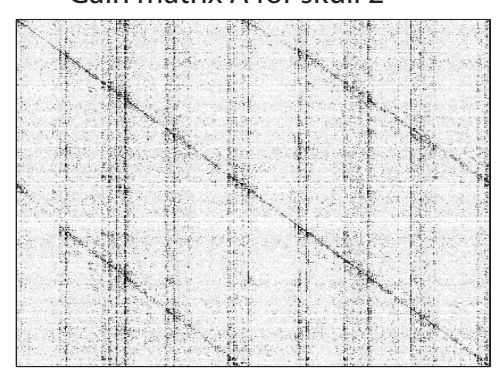

Gain matrix A for skull 5

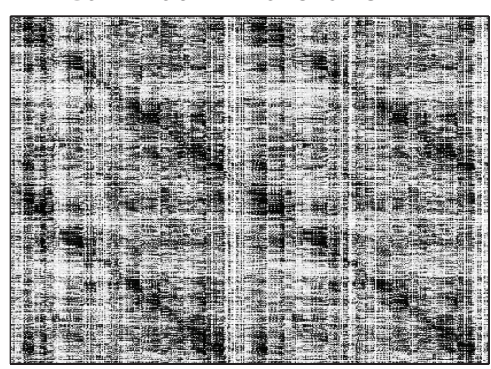

Gain matrix A for skull 3

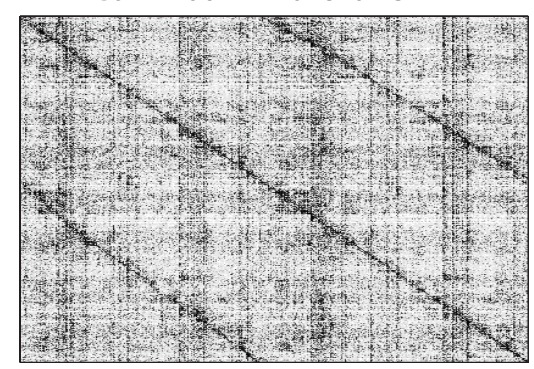

Gain matrix A for skull 6

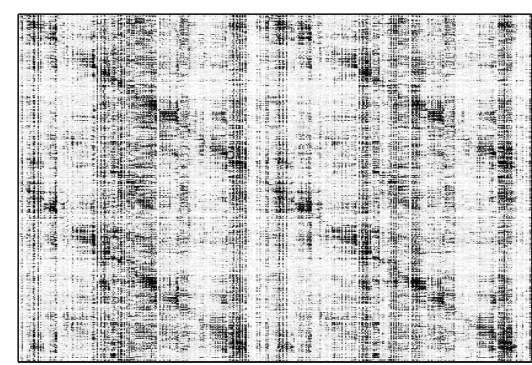

Figure 5. Actual gain matrices $A^{(s)}$ obtained experimentally for $S=6$ human skulls. Plots are normalized.

signal emitted by some transducers was more strongly scattered throughout the skull.

The singular values of these gain matrices $A^{(s)}$ are depicted in Fig. 6. As can be seen, for all the skulls, the singular values of $A^{(s)}$ decrease steadily, revealing an approximately low-rank structure. This suggests that good approximations of these matrices may be obtained by truncating the SVD to their first, say, 100 elements. This result is interesting because it was recently shown that approximate SVD may be computed efficiently for such matrices using randomized algorithms with a limited number of matrix-vector product evaluations [12]. Similarly, the calibration procedure proposed here aims at estimating approximations of $A$ using only a limited number of measurements.

4.2.2. Focusing The focusing results are displayed in Fig. 7, which is the main result of this paper. As can be seen, all the considered calibration techniques yield approximately similar results whenever $K>200$, meaning that when $X$ spans a sufficiently large subspace of $\mathbb{C}^{N}$, the calibration can be achieved using $K<N$ independent measurements. Interestingly, the typical $K$ required for good focusing is related to the number of large singular values of the gain matrices $A^{(s)}$ (as shown in Fig. 6), since we see that $90 \%$ focusing efficiency can be achieved using as few as $K=150$ calibration measures, for some choices of calibration signals. The use of a particular input scheme such as the Zernike polynomials, Hadamard, or random draws does not appear to be of a particular importance for $K>200$.

However, the use of a randomized scheme was seen to consistently outperform any deterministic method at lower $K$. In particular, the randomized methods $(i i)$ and (iii) 


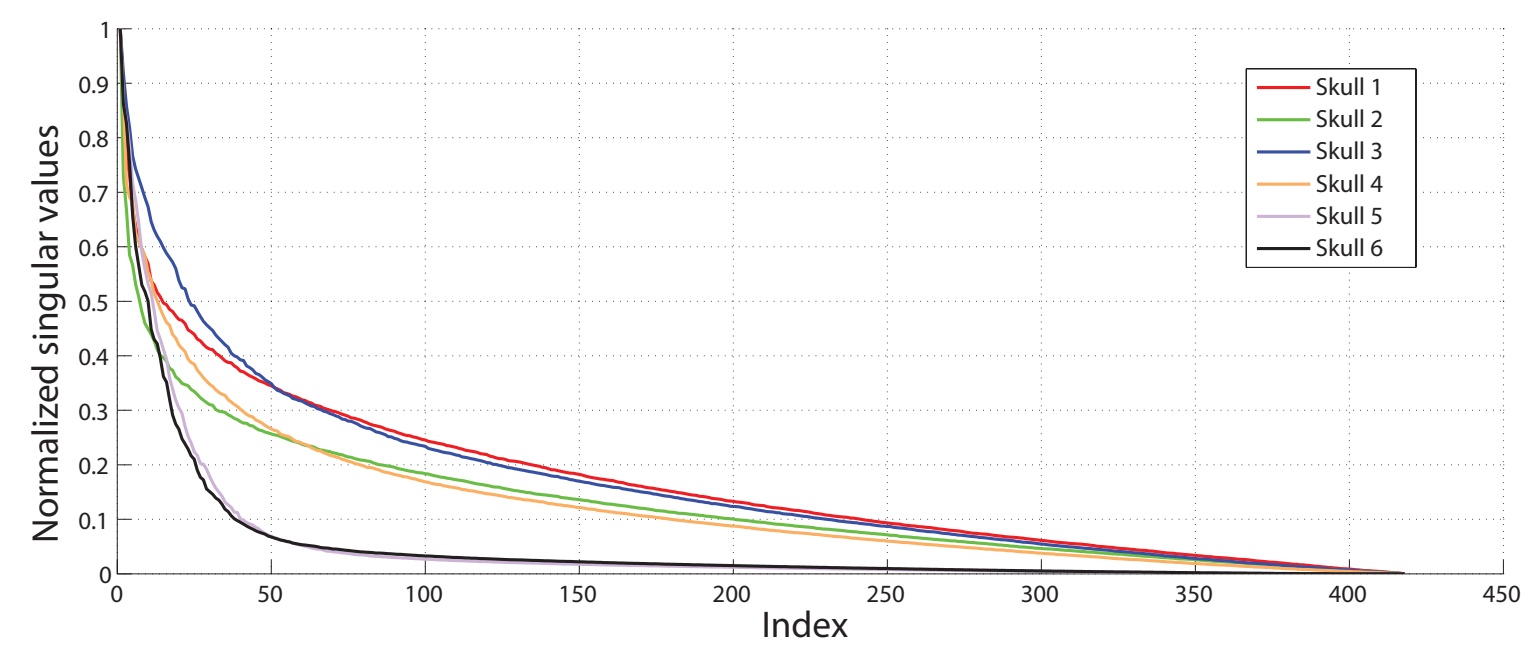

Figure 6. The singular values of the skull gain matrices $A^{(s)}$.

give a $10 \%$ focusing improvement over the Zernike or Hadamard schemes at small $K$. We explain this result by two facts. First, since they involve all transducers emitting at full power for each measurement, they provide an increase in the signal to noise ratio. Second, the use of a randomized scheme is optimal for the purpose of spanning an unknown subspace of $\mathbb{C}^{N}$ [12]. Interestingly, these randomized schemes performed as well as the "oracle" reference method (vii), which uses the actual eigenvectors of $A$.

An example of simulated focusing at a given voxel $m$ for different numbers $K$ of calibration measurements is displayed in Fig. 8, with a random $X_{n k} \in\{0,1\}$ scheme. This example concerns skull $s=1$, whose SVD decreases the most slowly, making it the most difficult example. As can also be seen in this figure, the focusing was already good with $K=75$, and reached $90 \%$ of its maximal efficiency for $K=150$. For $K>150$, no significant difference in the focusing pattern can be seen.

\section{Discussion}

\subsection{Randomized schemes for accelerating HIFU calibration}

In this study, we have demonstrated with both simulations and actual measurements gathered from six human skulls that it was possible to drastically reduce the number of measurements required for the calibration of HIFU for non-invasive transcranial therapy.

Our model exploits prior knowledge of the free-field transmission matrix (TM) $P_{0}$ that encodes the linear relation between the signal emitted by the $N$ transducers and the observed output in the focusing region of interest (ROI). The measurement of the freefield TM depends on the position of the transducers but does not require the presence of the patient as long as the actual target relative to the transducer array belongs to the ROI. The measurement of this matrix can be performed once for all, without restrictions concerning the acquisition time. Once this is done, the same matrix can be used for 


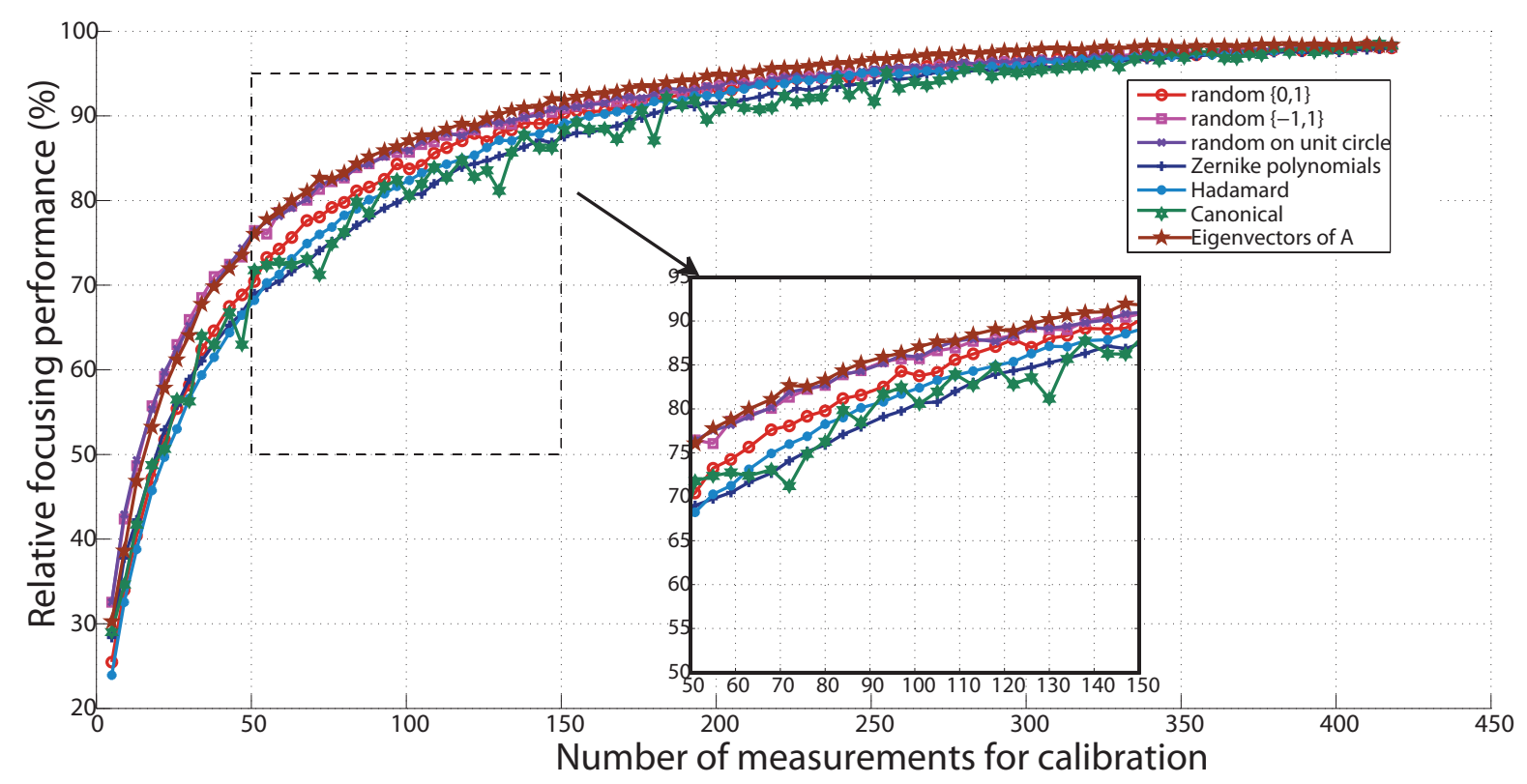

Figure 7. Focusing performance for 7 different calibration methods, corresponding to different choices of the calibration input matrix $X \in \mathbb{C}^{N \times K}$.

all subsequent skulls. Then, the actual TM $P$ that relates the input transducers to displacements in the ROI is modeled by $P=P_{0} A$, where $A$ is a gain matrix that can be understood as encapsulating all the acoustic aberrations induced by the skull. We provide a principled way to estimate this matrix given only a limited number $K<N$ of calibration measurements.

As shown, the input signal to be emitted by the transducers during calibration may be set in a randomized way and this choice leads to a focusing performance that consistently outperforms deterministic schemes, such as Hadamard [22] or Zernike polynomials [20]. In practice, we have shown that efficient focusing can be achieved with typically $K=N / 3$ calibration measurements. In terms of the focusing performance, the randomized approaches outperform the deterministic ones by approximately $10 \%$ for $K=75$. The choice of a randomized approach for calibration has the great advantage of being easily implementable, without requiring any knowledge of the actual spatial position of each transducer.

\subsection{Linearity of the model}

The method described in this paper assumes a linear propagation of the ultrasonic wave in tissues and through the skull [41]. But nonlinearities are known to develop along the propagation of a wave in tissue [45]. Nevertheless, this is neglected in current CT-based correction as well, as used during treatment planning for MR guided transcranial brain therapy $[6,19,24]$. Phase aberration correction techniques in general assume a linear propagation [40]. Nonlinear effects during the transcranial propagation of high intensity ultrasonic waves have been shown to be mostly confined to the vicinity of the focus [34]: 


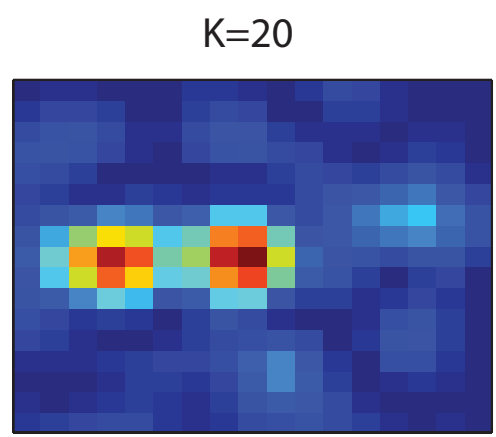

$\mathrm{K}=50$

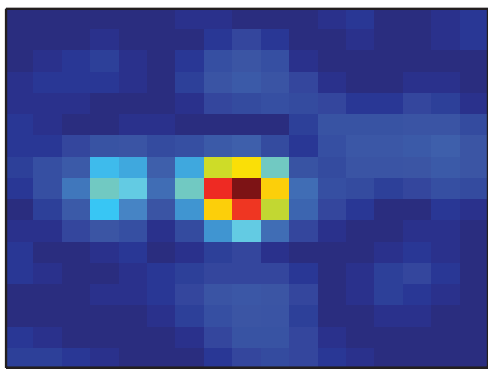

$\mathrm{K}=150$

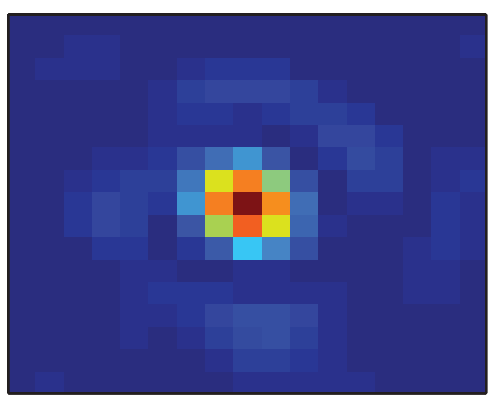

$\mathrm{K}=30$

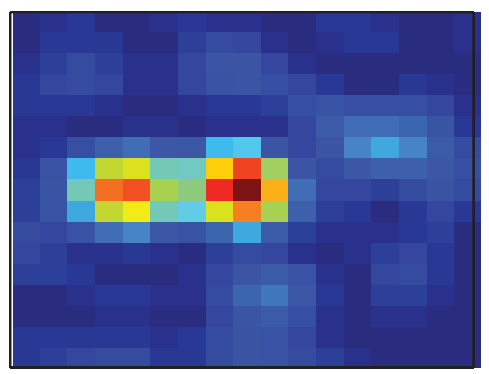

$\mathrm{K}=75$

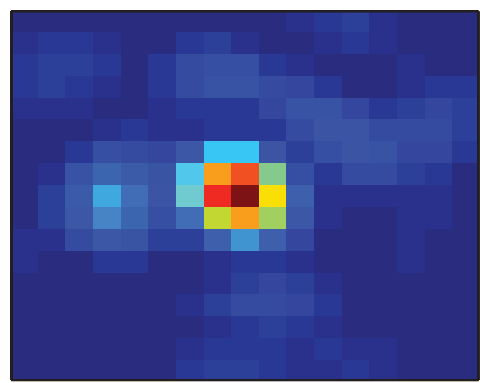

$K=200$

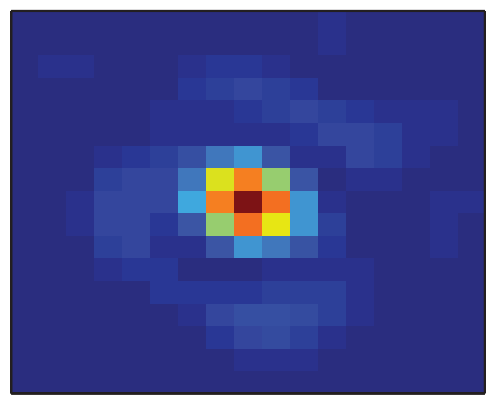

$\mathrm{K}=40$

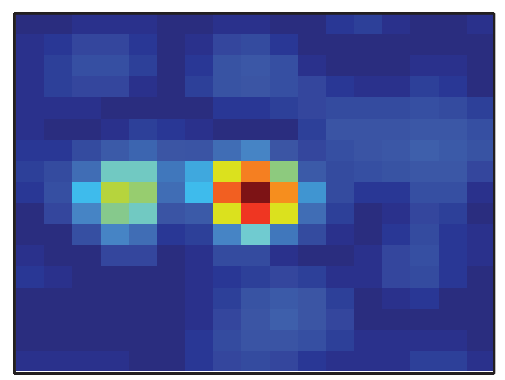

$K=100$

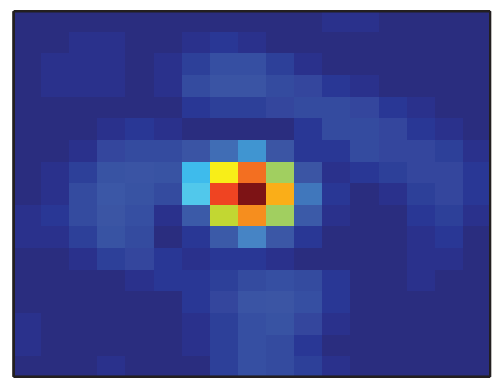

$\mathrm{K}=418$

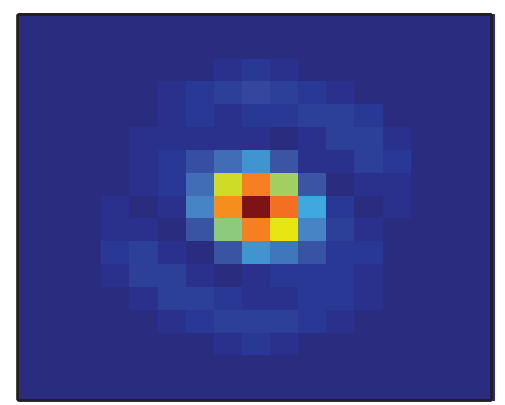

Figure 8. Examples of simulated focusing through skull $s=1$ for different numbers $K$ of random $X_{n k} \in\{0,1\}$ calibration measurements. Plots are normalized.

the skull mainly acts as a low-pass filter that resets the nonlinearities developing between the array of transducers and the skull.

\subsection{Future research}

This research may be continued along several directions.

First and foremost, in this proof-of-concept study, the experimental data was gathered from human skulls using a transcranial HIFU device in a water tank, and therefore the measurement of the TM was invasive. Future research will include noninvasive MR-ARFI experiments using the proposed randomized calibration schemes.

Second, the proposed randomized approach makes no assumption whatsoever concerning the geometry of the skull, nor of that of the transducer array. As [20] demonstrated, exploiting such prior knowledge definitely improves the performance over blind approaches. Hence, a further increase of performance is possible if the random 
drawing of the calibration configurations $X$ does not uniformly span $\mathbb{C}^{N}$, but would be rather mainly concentrated on the eigenvectors of $A$, which are supposed known a priori. This knowledge may for instance come from measurements taken on other subjects.

Finally, we plan to further simplify the calibration procedure by avoiding the phasestepping technique $[22,20,43]$ that is required to measure $y=P x$ with MRI hardware. Indeed, MRI actually measures $|y|^{2}$ only. Different measurements with varying input phases are required to derive $y$. However, recent research [31] has demonstrated that intensity-based signal reconstruction is feasible using dedicated phase-retrieval algorithms.

\section{Acknowledgements}

The authors would like to thank Benoit Larrat for valuable discussions. N. Liu would like to acknowledge the financial support from the China Scholarship Council (CSC). L. Daudet acknowledges support from Institut Universitaire de France. ESPCI ParisTech is part of the PSL Research University. This work was supported by the Agence Nationale de la Recherche under the program "Future Investments" with the reference ANR-10EQPX-15 and by LABEX WIFI (Laboratory of Excellence ANR-10-LABX-24) within the French Program "Investments for the Future" under reference ANR-10- IDEX-000102 PSL $\star$.

\section{References}

[1] J F Aubry, M Tanter, J Gerber, J L Thomas, and M Fink. Optimal focusing by spatio-temporal inverse filter. II. Experiments. Application to focusing through absorbing and reverberating media. The Journal of the Acoustical Society of America, 110(1):48-58, 2001.

[2] J F Aubry, M Tanter, M Pernot, J L Thomas, and M Fink. Experimental demonstration of noninvasive transskull adaptive focusing based on prior computed tomography scans. The Journal of the Acoustical Society of America, 113(1):84-93, 2003.

[3] D Chauvet, L Marsac, M Pernot, A-L Boch, R Guillevin, N Salameh, L Souris, L Darrasse, M Fink, M Tanter, et al. Targeting accuracy of transcranial magnetic resonance-guided high-intensity focused ultrasound brain therapy: A fresh cadaver model: Laboratory investigation. Journal of Neurosurgery, 118(5):1046-1052, 2013.

[4] G T Clement and K Hynynen. Correlation of ultrasound phase with physical skull properties. Ultrasound in Medicine \& Biology, 28(5):617-624, 2002.

[5] W J Elias, D Huss, T Voss, J Loomba, M Khaled, E Zadicario, R C Frysinger, S A Sperling, S Wylie, S J Monteith, et al. A pilot study of focused ultrasound thalamotomy for essential tremor. New England Journal of Medicine, 369(7):640$648,2013$. 
[6] W J Elias, M Khaled, J D Hilliard, J-F Aubry, R C Frysinger, J P Sheehan, $\mathrm{M}$ Wintermark, and M B Lopes. A magnetic resonance imaging, histological, and dose modeling comparison of focused ultrasound, radiofrequency, and Gamma Knife radiosurgery lesions in swine thalamus: Laboratory investigation. Journal of Neurosurgery, 119(2):307-317, 2013.

[7] F M Fennessy and C M Tempany. A review of magnetic resonance imaging-guided focused ultrasound surgery of uterine fibroids. Topics in Magnetic Resonance Imaging, 17(3):173-179, 2006.

[8] J L Foley, M Eames, J Snell, A Hananel, N Kassell, and J-F Aubry. Image-guided focused ultrasound: State of the technology and the challenges that lie ahead. Imaging in Medicine, 5(4):357-370, 2013.

[9] F J Fry and J E Barger. Acoustical properties of the human skull. The Journal of the Acoustical Society of America, 63(5):1576-1590, 1978.

[10] J Gâteau, L Marsac, M Pernot, J-F Aubry, M Tanter, and M Fink. Transcranial ultrasonic therapy based on time reversal of acoustically induced cavitation bubble signature. IEEE Transactions on Biomedical Engineering, 57(1):134-144, 2010.

[11] A Goetschy and AD Stone. Filtering random matrices: The effect of incomplete channel control in multiple scattering. Physical Review Letters, 111(6):063901, 2013.

[12] N Halko, P-G Martinsson, and J A Tropp. Finding structure with randomness: Probabilistic algorithms for constructing approximate matrix decompositions. SIAM review, 53(2):217-288, 2011.

[13] E Herbert, M Pernot, G Montaldo, M Fink, and M Tanter. Energy-based adaptive focusing of waves: Application to noninvasive aberration correction of ultrasonic wavefields. IEEE Transactions on Ultrasonics, Ferroelectrics and Frequency Control, 56(11):2388-2399, 2009.

[14] A B Holbrook, P Ghanouni, J M Santos, Y Medan, and K B Pauly. In vivo MR acoustic radiation force imaging in the porcine liver. Medical Physics, 38(9):50815089, 2011.

[15] M Huisman and MAAJ van den Bosch. MR-guided high-intensity focused ultrasound for noninvasive cancer treatment. Cancer Imaging, 11(1A):S161, 2011.

[16] K Hynynen and J Sun. Trans-skull ultrasound therapy: The feasibility of using image-derived skull thickness information to correct the phase distortion. IEEE Transactions on Ultrasonics, Ferroelectrics and Frequency Control, 46(3):752-755, 1999.

[17] M E Ikink, M J Voogt, H M Verkooijen, P NM Lohle, K J Schweitzer, A Franx, P Th M Willem, L W Bartels, and M AAJ van den Bosch. Mid-term clinical efficacy of a volumetric magnetic resonance-guided high-intensity focused ultrasound technique for treatment of symptomatic uterine fibroids. European Radiology, 23(11):3054-3061, 2013. 
[18] R O Illing, J E Kennedy, F Wu, G R ter Haar, A S Protheroe, P J Friend, F V Gleeson, D W Cranston, R R Phillips, and M R Middleton. The safety and feasibility of extracorporeal high-intensity focused ultrasound (HIFU) for the treatment of liver and kidney tumours in a Western population. British Journal of Cancer, 93(8):890-895, 2005.

[19] D Jeanmonod, B Werner, A Morel, L Michels, E Zadicario, G Schiff, and E Martin. Transcranial magnetic resonance imaging-guided focused ultrasound: noninvasive central lateral thalamotomy for chronic neuropathic pain. Neurosurgical Focus, 32(1):E1, 2012.

[20] E A Kaye, Y Hertzberg, M Marx, B Werner, G Navon, M Levoy, and K B Pauly. Application of Zernike polynomials towards accelerated adaptive focusing of transcranial high intensity focused ultrasound. Medical Physics, 39(10):6254-6263, 2012.

[21] B Larrat, M Pernot, J-F Aubry, E Dervishi, R Sinkus, D Seilhean, Y Marie, A-L Boch, M Fink, and M Tanter. MR-guided transcranial brain HIFU in small animal models. Physics in Medicine and Biology, 55(2):365, 2010.

[22] B Larrat, M Pernot, G Montaldo, M Fink, and M Tanter. MR-guided adaptive focusing of ultrasound. IEEE Transactions on Ultrasonics, Ferroelectrics and Frequency Control, 57(8):1734-1747, 2010.

[23] B Liberman, D Gianfelice, Y Inbar, A Beck, T Rabin, N Shabshin, G Chander, $\mathrm{S}$ Hengst, R Pfeffer, A Chechick, et al. Pain palliation in patients with bone metastases using MR-guided focused ultrasound surgery: A multicenter study. Annals of Surgical Oncology, 16(1):140-146, 2009.

[24] N Lipsman, M L Schwartz, Y Huang, L Lee, T Sankar, M Chapman, K Hynynen, and A M Lozano. MR-guided focused ultrasound thalamotomy for essential tremor: A proof-of-concept study. The Lancet Neurology, 12(5):462-468, 2013.

[25] F Marquet, M Pernot, JF Aubry, G Montaldo, L Marsac, M Tanter, and M Fink. Non-invasive transcranial ultrasound therapy based on a 3D CT scan: Protocol validation and in vitro results. Physics in Medicine and Biology, 54(9):2597, 2009.

[26] L Marsac, D Chauvet, B Larrat, M Pernot, B Robert, M Fink, A-L Boch, J-F Aubry, and M Tanter. MR-guided adaptive focusing of therapeutic ultrasound beams in the human head. Medical Physics, 39(2):1141-1149, 2012.

[27] N McDannold and S E Maier. Magnetic resonance acoustic radiation force imaging. Medical Physics, 35(8):3748-3758, 2008.

[28] C A Molina, A D Barreto, G Tsivgoulis, P Sierzenski, M D Malkoff, M Rubiera, N Gonzales, R Mikulik, G Pate, J Ostrem, et al. Transcranial ultrasound in clinical sonothrombolysis (TUCSON) trial. Annals of Neurology, 66(1):28-38, 2009.

[29] F Murat, L Poissonnier, G Pasticier, and A Gelet. High-intensity focused ultrasound (HIFU) for prostate cancer. Cancer Control, 14(3):244, 2007. 
[30] A Napoli, M Anzidei, B C Marincola, G Brachetti, F Ciolina, G Cartocci, C Marsecano, F Zaccagna, L Marchetti, E Cortesi, et al. Primary pain palliation and local tumor control in bone metastases treated with magnetic resonance-guided focused ultrasound. Investigative Radiology, 48(6):351-358, 2013.

[31] H Ohlsson, A Y Yang, R Dong, and S S Sastry. Compressive phase retrieval from squared output measurements via semidefinite programming. arXiv preprint arXiv, 1111, 2011.

[32] M Pernot, J F Aubry, M Tanter, A L Boch, F Marquet, M Kujas, D Seilhean, and M Fink. In vivo transcranial brain surgery with an ultrasonic time reversal mirror. Journal of Neurosurgery, 106(6):1061-1066, 2007.

[33] G Pinton, J F Aubry, E Bossy, M Muller, M Pernot, and M Tanter. Attenuation, scattering, and absorption of ultrasound in the skull bone. Medical Physics, 39(1):299-307, 2011.

[34] G Pinton, J F Aubry, M Fink, and M Tanter. Effects of nonlinear ultrasound propagation on high intensity brain therapy. Medical Physics, 38(3):1207-1216, 2011.

[35] S Popoff, G Lerosey, M Fink, A C Boccara, and S Gigan. Image transmission through an opaque material. Nature Communications, 1:81, 2010.

[36] SM Popoff, G Lerosey, M Fink, A C Boccara, and S Gigan. Controlling light through optical disordered media: Transmission matrix approach. New Journal of Physics, 13(12):123021, 2011.

[37] R Sinkus, M Tanter, J Bercoff, K Siegmann, M Pernot, A Athanasiou, and M Fink. Potential of MRI and ultrasound radiation force in elastography: Applications to diagnosis and therapy. Proceedings of the IEEE, 96(3):490-499, 2008.

[38] E A Stewart, J Rabinovici, C Tempany, Y Inbar, L Regan, B Gastout, G Hesley, H S Kim, S Hengst, and W M Gedroye. Clinical outcomes of focused ultrasound surgery for the treatment of uterine fibroids. Fertility and Sterility, 85(1):22-29, 2006.

[39] J Sun and K Hynynen. The potential of transskull ultrasound therapy and surgery using the maximum available skull surface area. The Journal of the Acoustical Society of America, 105(4):2519-2527, 1999.

[40] M Tanter, J F Aubry, J Gerber, J L Thomas, and M Fink. Optimal focusing by spatio-temporal inverse filter. I. Basic principles. Journal of the Acoustical Society of America, 110(1):37-47, 2001.

[41] M Tanter, J L Thomas, and M Fink. Time reversal and the inverse filter. The Journal of the Acoustical Society of America, 108(1):223-234, 2000.

[42] J L Thomas and M A Fink. Ultrasonic beam focusing through tissue inhomogeneities with a time reversal mirror: Application to transskull therapy. IEEE Transactions on Ultrasonics, Ferroelectrics and Frequency Control, 43(6):1122-1129, 1996. 
[43] S Tripathi, R Paxman, T Bifano, and K C Toussaint. Vector transmission matrix for the polarization behavior of light propagation in highly scattering media. Optics Express, 20(14):16067-16076, 2012.

[44] F Wu, Z B Wang, W Z Chen, W Wang, Y Gui, M Zhang, G Zheng, Y Zhou, $\mathrm{G} \mathrm{Xu}, \mathrm{M} \mathrm{Li}$, et al. Extracorporeal high intensity focused ultrasound ablation in the treatment of 1038 patients with solid carcinomas in China: An overview. Ultrasonics Sonochemistry, 11(3):149-154, 2004.

[45] R J Zemp, J Tavakkoli, and R SC Cobbold. Modeling of nonlinear ultrasound propagation in tissue from array transducers. The Journal of the Acoustical Society of America, 113(1):139-152, 2003. 